\title{
Un análisis semiótico del objeto tabla estadística en libros de texto chilenos
}

\author{
A semiotic analysis of the object statistical table in Chilean textbooks
}

\author{
Jocelyn D. Pallauta ${ }^{1}$ \\ María M. Gea ${ }^{2}$ \\ Carmen Batanero ${ }^{3}$
}

\begin{abstract}
Resumen
En este artículo se realiza un análisis semiótico de los objetos matemáticos primarios en el trabajo con diferentes tipos de tablas estadísticas, utilizando elementos del enfoque ontosemiótico. Dicho análisis, se utiliza para extender los niveles de complejidad semiótica definidos por Arteaga para los gráficos estadísticos a las tablas estadísticas. También se aborda, a través de un análisis de contenido, la distribución de estos tipos de tabla en una muestra de doce libros escolares chilenos, dirigidos de $5^{\circ}$ a $8^{\circ}$ Básico (10 a 13 años). La representación tabular con mayor presencia en estos textos es la tabla de distribución, que se enmarcaría en un nivel 3 de complejidad semiótica, subdividido en tres niveles, y dentro de ellos el más frecuente es el primero, apareciendo el último sólo en $8^{\circ}$ curso. La tabla de contingencia, asociada al máximo nivel de complejidad, aparece con poca frecuencia y paradójicamente disminuye su uso con el curso escolar.
\end{abstract}

Palabras clave: Tablas estadísticas; análisis semiótico; niveles de complejidad semiótica; libros de texto.

\begin{abstract}
In this paper we perform a semiotic analysis of the primary mathematical objects implicit in the work with different types of statistical tables, using elements of the ontosemiotic approach. This analysis, is used to extend the levels of semiotic complexity defined by Arteaga for statistical graphs to statistical tables. The distribution of these types of tables in a sample of twelve Chilean school books, directed from 5th to 8th grade (10 to 13 years) is also presented through a content analysis. The tabular representation with greater presence in these texts is the distribution table, which corresponds to level 3 of semiotic complexity, subdivided into three levels, and within them the most frequent is the first, since the last sublevel only appears in 8th grade. The contingency table, associated with the maximum level of complexity, appears scarcely and paradoxically its use decreases with the school year.
\end{abstract}

Keywords: Statistical tables; semiotic analysis; level of semiotic complexity; textbooks.

\section{Introducción}

Submetido em: 18/08/2019 - Aceito em: 06/01/2020 - Publicado em: 16/01/2020

1 Máster en Educación Matemática, Universidad de Granada, España. Correo electrónico: jocelyndiaz@correo.ugr.es.

${ }^{2}$ Doctorado en Educación, Universidad de Granada. Ayudante de docencia en la Universidad de Granada, España. Correo electrónico: mmgea@ugr.es.

3 Doctorado en Matemáticas, Universidad de Granada. Profesor de Educación Matemática, Universidad de Granada, España. Correo electrónico: batanero@ugr.es. 
El currículo escolar de distintos países ha incorporado el estudio de la estadística desde temprana edad (e.g. MECD, 2014; MINEDUC, 2015; 2018), reconociendo su importancia en la formación de los ciudadanos, destacada por varios autores como medio de comprensión de nuestra sociedad (Engel, 2019; Gal, 2002). En estos lineamientos curriculares, se destaca la necesidad de que los estudiantes desarrollen habilidades en la representación e interpretación de datos, para reforzar su comprensión del mundo actual.

La tabla estadística, es un contenido importante en el estudio de la estadística como medio para representar y comunicar información (Autor, 2011). Feinberg y Wainer (2012) indican que su construcción e interpretación no son sencillas, pues requiere interpretar las variables y sus categorías (filas y columnas), además de comprender el tipo de información que se representa. Respecto a las mismas, se puede plantear una diversidad de tareas, como representar información, lectura, interpretación, o traducción entre representaciones (Koschat, 2005). Por otra parte, Estrella (2014) sugiere que en el proceso de enseñanza se prioriza más la técnica (pautas rutinarias), que la comprensión de aspectos fundamentales para su construcción o lectura.

Podemos encontrar una amplia variedad de tipos de tablas estadísticas, que fueron clasificadas en tres grupos principales por Lahanier-Reuter (2003). La finalidad de este trabajo es ampliar dicho estudio, analizando los objetos matemáticos implícitos en el trabajo con estos tipos de tabla. Adicionalmente, pretendemos adaptar el constructo complejidad semiótica, descrito por Arteaga (2011) para los gráficos estadísticos a las tablas estadísticas, y mostrar la diferente dificultad que implica el trabajo con cada una de ellas. Finalmente, analizamos la distribución de estos tipos de tablas en una muestra de textos chilenos de Educación Básica para proporcionar al profesor información detallada del nivel de complejidad requerido en el trabajo con las tablas estadísticas en los diferentes cursos donde se inicia su enseñanza.

\section{Fundamentos}

A continuación, incluimos los fundamentos del trabajo que son de dos tipos: el marco teórico y las investigaciones previas que conforman la base de nuestro análisis, para la posterior exposición de los resultados obtenidos en la investigación.

\section{Marco teórico}

Nos basamos en el enfoque ontosemiótico del conocimiento e instrucción matemática (Godino, 2002; 2017), en que la situación-problema y las prácticas que se realizan en su resolución permitirá definir el objeto matemático y su significado. Se diferencian dos dimensiones interdependientes del significado del objeto matemático (Autor, 2007; 2019): la dimensión personal (subjetiva, mental), y la dimensión institucional (objetiva, contextual). De acuerdo a este marco, el significado de la tabla estadística sería el conjunto de prácticas matemáticas realizadas para resolver problemas relacionados con ella.

En dichas prácticas, intervienen objetos matemáticos de distinta naturaleza: símbolos, gráficos, textuales, orales, entre otros. Si los objetos provienen de una institución se les 
DOI: $10.20396 /$ zet.v28i0.8656257

denominará "objetos institucionales" y si surgen de manera individual, "objetos personales". En Autor (2007; 2019) se proponen los siguientes tipos de objetos llamados primarios: lenguaje (términos, expresiones, notaciones, gráficos, etc.); situaciones-problemas (problemas, aplicaciones extra-matemáticas o matemáticas, ejercicios, etc.); conceptos, dados por su definición o descripción; proposiciones, propiedades o atributos; procedimientos (operaciones, algoritmos, técnicas); y argumentos o razonamientos usados para validar y explicar las proposiciones y procedimientos (deductivo, inductivo, etc.).

Además, tomaremos en cuenta los niveles de complejidad semiótica, que es un modelo propuesto por Arteaga y colaboradores (Arteaga, 2011; Autor, 2011; Autor, 2010). Estos autores definen cuatro niveles de complejidad en los gráficos, en función del tipo de objetos representados en ellos, pues la comprensión de los mismos requiere diferentes procesos interpretativos, tanto de cada elemento representado, como del gráfico en su conjunto. Los niveles propuestos son los siguientes:

- Nivel 1 (N1). Representar solo algunos datos aislados de una variable. En este nivel no se usa la idea de variable o de distribución.

- Nivel 2 (N2). Representar un conjunto de datos asociado a una variable, sin formar la distribución de frecuencias. Se utiliza la idea de variable y sus valores, pero no la de frecuencia o distribución.

- Nivel 3 (N3). Representar una distribución de frecuencias de una variable. En este caso, ya aparece el concepto de frecuencia y de distribución.

- Nivel 4 (N4). Representar una distribución de frecuencias de dos o más variables. Se emplean todos los objetos anteriores y además, generalmente, se utiliza una misma escala para representar las variables.

La finalidad de nuestro trabajo consiste en aplicar y ampliar estos niveles a las tablas estadísticas, cuya construcción, lectura e interpretación, también implica los objetos matemáticos descritos anteriormente.

\section{Investigaciones previas}

Además del marco teórico, tendremos en cuenta las investigaciones que analizan en forma teórica el objeto tabla estadística. Entre ellas, encontramos la desarrollada por Estrella (2014), quien indentifica los siguientes componentes en la tabla estadística:

- Título: debe describir las variables representadas, y si es posible, el contexto en que se realizó el estudio o la recogida de la información.

- Las etiquetas presentes en el margen lateral o primera columna: muestran las diferentes modalidades que toma la variable, de acuerdo a su escala de clasificación.

- Las etiquetas que se presentan en el encabezado superior o primera fila: donde se indica el contenido de cada una de las columnas. Por ejemplo, el tipo de frecuencias, o las modalidades de una segunda variable (tablas de doble entrada). Por otro lado, en la última fila y/o columna se ubican los totales.

- El cuerpo de los datos: es el conjunto de celdas que se forman al interior, como 
intersección de filas y columnas. Este contiene, generalmente, información numérica y puede ser de tipo diverso, como frecuencia absoluta o relativa, porcentaje e incluso, en algunos casos, puede contener más de un elemento en la misma celda.

Tipos de tablas

Lahanier-Reuter (2003) identifica tres tipos de tablas, presentes en la enseñanza de la estadística: tabla de datos, tabla de distribución de una variable, y la tabla de contingencia. Cada una posee funciones específicas que la dotan de sentido, y se analizan a continuación.

- Tabla de datos. Es la primera organización de un conjunto de datos. Tiene forma de matriz y contiene, para cada individuo de la muestra, los valores de una o varias variables.

- Tabla de distribución de una variable. Esta tabla se construye a partir del conjunto de datos sin clasificar o desde una tabla de datos. Describe la distribución de una variable, dado que asocia cada modalidad de la variable con el número de individuos de la muestra (frecuencia) que presentan dicha modalidad.

- Tabla de doble entrada o de contingencia. Representa datos mediante el cruce de dos variables estadísticas. En la parte superior de la tabla (primera fila), se indican las modalidades de una de las variables, mientras que, en la primera columna se indican las modalidades de la segunda variable. El cuerpo de la tabla está formado por las frecuencias conjuntas que corresponde a la modalidad de la fila para la primera variable y de la columna para la segunda variable.

Además, tendremos en cuenta las investigaciones de Díaz-Levicoy y colaboradores (Díaz-Levicoy, Morales, \& López-Martín, 2015), quienes analizan las tablas estadísticas en cuatro libros de texto chilenos de $1^{\circ}$ y $2^{\circ}$ curso de Educación Básica (6 y 7 años). Se considera, entre otras variables, el tipo de tabla (datos, conteo, frecuencia y doble entrada) en dos editoriales diferentes. En una de ellas, las tablas de conteo (un tipo de tabla en la que se registra los recuentos de frecuencia, a través de marcas o símbolos dentro de una celda) alcanzan alrededor de un $83 \%$, mientras que en la otra, el $42 \%$. En esta última, se muestra un reparto más equilibrado con tablas de frecuencias (50\%) y tablas de doble entrada (17\%), las que no son consideradas por la primera editorial. Este estudio, es complementado posteriormente (Díaz-Levicoy, Ruz, \& Molina-Portillo, 2017), analizando las tareas que abordan tablas estadísticas en tres libros de texto de tercer grado (8 años) de Educación Básica, también en Chile. El análisis mostró, que el tipo de tabla que aparece con mayor fuerza es la de conteo $(38,9 \%)$, seguida de la tabla de frecuencias $(32,2 \%)$, principalmente la absoluta, y luego las de datos $(25,6 \%)$, en menor medida aparecen tablas que combinan dos de los tipos mencionados anteriormente, como conteo y datos, o conteo y frecuencias. Llama la atención que, en este nivel educativo, la tabla de doble entrada no tuvo presencia en los textos explorados, a diferencia de lo ocurrido en el estudio dirigido a $1^{\circ}$ y $2^{\circ}$ curso (Díaz-Levicoy et al., 2015).

Nuestro trabajo completa los anteriores, al utilizar los niveles de complejidad semiótica y el análisis de los objetos matemáticos implícitos en los distintos tipos de tablas, para evidenciar que, a pesar de que visualmente puedan parecer muy similares, se requiere de 
diferentes demandas cognitivas, las que no siempre son explicitadas o abordadas por los profesores en la escuela (Koschat, 2005). Además, analizaremos la distribución de los tipos de tablas en los textos comparando con los estudios de Díaz-Levicoy y colaboradores, que fueron dirigidas para niveles educativos inferiores, también en Chile.

\section{Metodología}

La investigación que desarrollamos es de tipo descriptiva, y se emplea como técnica el análisis de contenido en una muestra de textos escolares, siguiendo etapas de modo sistemático (Mayring, 2000), lo que permite obtener categorías de análisis de manera cíclica e inductiva.

La muestra está constituida por doce textos escolares, publicados el año 2017 (Anexo), dirigidos a estudiantes de $5^{\circ}$ a $8^{\circ}$ curso (10 a 13 años) de Educación Básica de Chile, y atienden al marco curricular vigente (MINEDUC, 2015; 2018). Por cada nivel educativo se analizaron tres textos: el libro del estudiante, el cuaderno de ejercicios, y la guía didáctica para el docente. Cabe señalar, que los textos que componen la muestra se convierten en un recurso educativo primordial en la enseñanza y aprendizaje de las matemáticas en Chile, y fueron elegidos por ser los más utilizados, dado que son distribuidos de forma gratuita a los estudiantes del sistema público y particulares con aportes del Estado.

De dichos textos se ha realizado un análisis semiótico, a partir del estudio de las bases curriculares propuestas por el NCTM (2000), el proyecto GAISE (Franklin et al., 2005) y los lineamientos curriculares chilenos (MINEDUC, 2015; 2018), complementado también con libros especializados de Educación Estadística. Este proceso, permite valorar la instrucción planificada en un libro de texto, y su resultado se puede considerar como el significado institucional pretendido (Godino, 2002; 2017) para los niveles educativos examinados.

Para llevar a cabo el análisis se estudia, para cada tipo de tabla, los elementos primarios considerados en el enfoque ontosemiótico, pues el trabajo con la tabla estadística requiere activar un conjunto de elementos que conforma una configuración epistémica, en cada una de ellas (Godino, 2002; 2017), compuesta de los siguientes objetos: situaciones-problemas, lenguaje, argumentos y reglas (conceptos, proposiciones y procedimientos).

\section{Resultados}

Presentamos a continuación, los resultados del análisis: En primer lugar, describimos los niveles de complejidad semiótica correspondientes a los diferentes tipos de tabla estadística descritos por Lahanier-Reuter (2003), para adaptar a las mismas los niveles de complejidad semiótica definidos para los gráficos estadísticos por Arteaga (2011). Seguidamente, para cada tipo de objeto primario considerado en el marco teórico EOS (Godino, 2002; 2017), describimos los asociados a cada tipo de tabla. Se finaliza con un estudio de la distribución de los tipos de tablas en los textos de la muestra.

Niveles de complejidad semiótica asociados a diferentes tipos de tablas 
Los niveles de complejidad semiótica, definidos para los gráficos estadísticos por Arteaga y colaboradores (Arteaga, 2011; Autor, 2011; Autor, 2010), pueden ser adaptados al trabajo con las tablas estadísticas. Para ello, necesitamos ajustar su categorización, pues no se contempla el nivel más bajo y, además, es necesario subdividir los dos niveles superiores en otros. Dichos niveles guardan una estrecha relación con los diferentes tipos de tablas descritos por Lahanier-Reuter (2003):

- Tabla de datos. Se caracterizan por su gran tamaño, dado que poseen tantas filas como individuos en la muestra. Generalmente, en la parte superior de la tabla, se incluye un encabezado con la descripción de las variables, recogidas en cada individuo. La primera columna de la izquierda contiene el listado con los sujetos, mientras que en cada una de las siguientes columnas aparecen el listado de los datos asociados a cada variable en cada uno de ellos. En ocasiones, las variables aparecen obedeciendo cierta jerarquía u orden; por ejemplo, en la Figura 1, los individuos son fechas que aparecen ordenadas cronológicamente, en las cuáles se ha recogido datos en dos años. En nuestra propuesta, este tipo de tabla corresponde al nivel semiótico N2, descrito por Arteaga (2011), puesto que en esta representación aparece la idea de variable y valor de la variable, pero no se muestran frecuencias asociadas a cada modalidad y, por tanto, no emerge el concepto de distribución de la variable estadística.

b leen e interpretan la información correspondiente a las tempe-
raturas máximas de los diez orimeros dias del mes de octubre
en los años 2009 y 2010
\begin{tabular}{lll}
\hline & 2009 & 2010 \\
\hline $01 / 10 / 2010$ & $22^{\circ}$ & $24^{\circ}$ \\
\hline $02 / 10 / 2010$ & $25^{\circ}$ & $25^{\circ}$ \\
\hline $03 / 10 / 2010$ & $26^{\circ}$ & $20^{\circ}$ \\
\hline $04 / 10 / 2010$ & $21^{\circ}$ & $18^{\circ}$ \\
\hline $05 / 10 / 2010$ & $18^{\circ}$ & $19^{\circ}$ \\
\hline $06 / 10 / 2010$ & $20^{\circ}$ & $21^{\circ}$ \\
\hline $07 / 10 / 2010$ & $25^{\circ}$ & $18^{\circ}$ \\
\hline $08 / 10 / 2010$ & $26^{\circ}$ & $20^{\circ}$ \\
\hline
\end{tabular}

Figura 1 - Listado de datos propuesto para $5^{\circ}$ Básico

Fuente: MINEDUC (2013, p.147)

- Tabla de distribución de una variable. Habitualmente, este tipo de tabla posee varias columnas. Generalmente, en la parte superior (primera fila) se indican el tipo de frecuencias representadas en la tabla (absolutas, relativas, acumuladas o porcentajes). En la primera columna se indican las diferentes modalidades o valores que toma la variable. Y en cada una de las siguientes columnas, las celdas de la tabla contienen frecuencia que corresponde a dicho valor o modalidad (del tipo indicado en la primera fila para esa columna). En ocasiones, la última fila es destinada para registrar valores totales, según sea el interés. Si el número de valores de la variable o categorías es pequeño, se puede intercambiar filas y columnas en la tabla.

Un ejemplo tomado de un libro de texto se muestra en la Figura 2. En esta tabla, además 
de la frecuencia absoluta, se incluye la frecuencia acumulada, y se añade una fila con el cálculo de cuartiles. Sugerimos que estas tablas corresponden al nivel semiótico N3 (Arteaga, 2011), puesto que en ella se representa la distribución de frecuencias de una variable. Este nivel, además, podríamos dividirlo en tres subniveles, dependiendo de si se consideran las frecuencias acumuladas y los intervalos de clase. Su clasificación obedece a los objetos matemáticos que en ellas intervienen (Autor, 2000), dotándolas de diferente sentido y complejidad para el estudiante.

- Nivel de complejidad N3.1: Tablas de distribución de frecuencias ordinarias: absolutas, relativas o porcentuales.

- Nivel de complejidad N3.2: Tablas de distribución de frecuencias, que incluyen también frecuencias acumuladas (absolutas, relativas o porcentuales). Su nivel de complejidad es mayor, porque involucra el manejo de desigualdades.

- Nivel de complejidad N3.3: Cuando se considera la agrupación de los valores de la variable en intervalo, para cualquier tipo de frecuencia, tanto ordinaria como acumulada. Se añade la idea de intervalo, sus extremos y marca de clase.

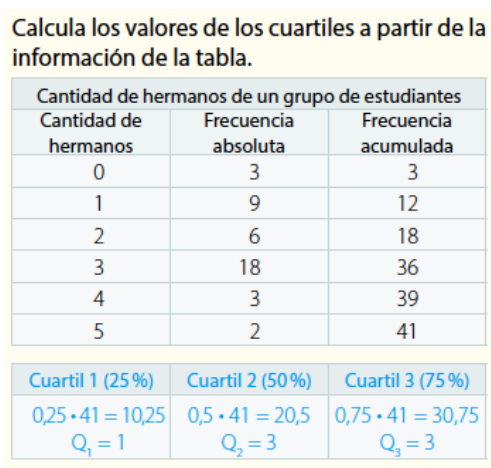

Figura 2 - Tabla de distribución para $8^{\circ}$ Básico Fuente: Maldonado, \& Castro (2017, p. 317)

- Tabla de contingencia. Como en el caso anterior, las frecuencias de las celdas pueden ser ordinarias (absolutas, relativas o porcentuales), pero en este caso, encontramos diferentes tipos de frecuencias relativas (respecto a la fila o a la columna). La última fila y la última columna son destinadas a registrar subtotales, de filas y columnas respectivamente. Incluimos en la Figura 3 un ejemplo para $7^{\circ}$ curso (12 años). Estas tablas corresponderían al nivel más alto de complejidad semiótica: N4 según Arteaga (2011), pues permiten comparar la distribución de dos variables estadísticas unidimensionales y relacionarlas entre sí. Estas tablas, a su vez, podrían contener intervalos de clase, aunque no es habitual considerar frecuencias acumuladas. Por tanto, se podría clasificar este nivel en dos subniveles, según se consideren intervalos de clase o no:

- Nivel de complejidad N4.1: Tablas de contingencia de frecuencias ordinarias: absolutas, relativas o porcentuales.

- Nivel de complejidad N4.2: Cuando se considera la agrupación de los valores de la variable en intervalo, para cualquier tipo de frecuencia. 
DOI: $10.20396 /$ zet.v28i0.8656257

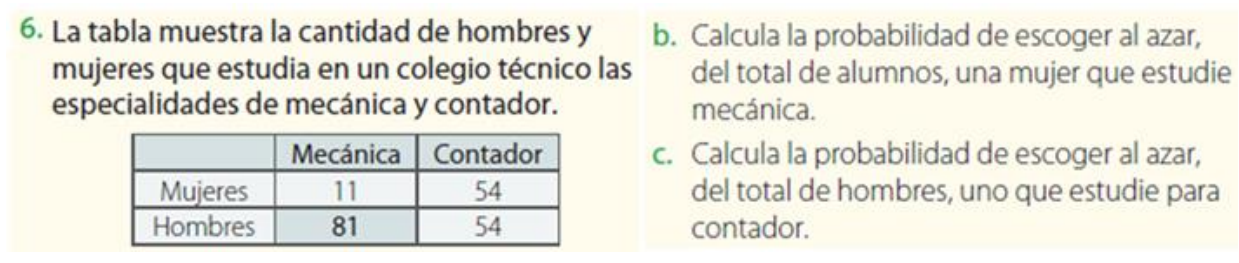

Figura 3 - Tabla de contingencia para $7^{\circ}$ Básico

Fuente: Merino, Muñoz, Pérez, \& Rupin (2016, p. 348)

Una vez propuesta nuestra clasificación de las tablas, según niveles de complejidad semiótica, se procede al análisis semiótico de las mismas, considerando sucesivamente los diferentes tipos de objetos matemáticos ligados al trabajo con las tablas estadísticas.

\section{Situaciones-problemas ligadas al trabajo con las tablas estadísticas}

En el EOS, la situación-problema tiene un rol relevante, pues los objetos matemáticos intervienen y emergen de las prácticas, personales o institucionales, al resolver problemas (Autor, 2007; 2019). Se han encontrado en los textos las siguientes situaciones-problemas (SP):

- SP1. El primero de estos problemas es la organización de los datos, que conlleva el registro de los mismos. Se produce cuando se recogen datos con algún propósito y es necesario organizarlos para interpretarlos. Como Estrella (2014) sugiere, este uso es muy antiguo y se ha encontrado en diferentes civilizaciones. Un ejemplo se muestra en la Figura 1.

- SP2. Otro problema diferente sería la construcción de la distribución de una variable estadística a través de la tabla, que conlleva el cálculo de la frecuencia de cada una de sus modalidades y su organización en la tabla de distribución (Ver Figura 2). Involucra la acción de organizar un conjunto de datos, que pueden ser entregados o recolectados por el propio estudiante, asociados a una variable estadística unidimensional, en una tabla en que se presenten una o varios tipos de frecuencias (ordinarias o acumuladas). Aparece con la necesidad de resumir un listado de datos, teniendo en cuenta que los mismos valores de la variable se repiten en el listado. Se podrían diferenciar subproblemas, según el tipo de variable, si se trabaja con más de una o según las frecuencias que se desea calcular. Dependiendo de la extensión del listado de datos, en algunos casos, es necesario distribuir las modalidades de la variable por medio de la agrupación en intervalos.

- SP3. Traducción entre representaciones. Este tipo de situación-problema lo dividimos en cuatro categorías, para diferenciar cada uno de los procesos de traducción vinculados a la tabla estadística: de tabla a gráfico o viceversa, de tabla a tabla, de tabla a texto o viceversa, y de tabla a estadístico. Un ejemplo de traducción de gráfico a tabla se presenta en la Figura 4. Dicha traducción puede implicar un proceso de transnumeración (Wild, \& Pfannkuch, 1999), al permitir visualizar nueva información desde los datos. Este cambio de representación de la información y su posterior análisis involucra una serie de tareas tales como: distinguir y clasificar datos, cálculo de 
medidas de tendencia central o dispersión en una variable, cálculo de frecuencia de datos, entre otras. En el procedimiento de transnumeración con las tablas estadísticas, Chick (2004) identifica diferentes etapas previas al proceso, como representar los datos en tablas o gráficos, el cálculo de frecuencias de datos, entre otros.

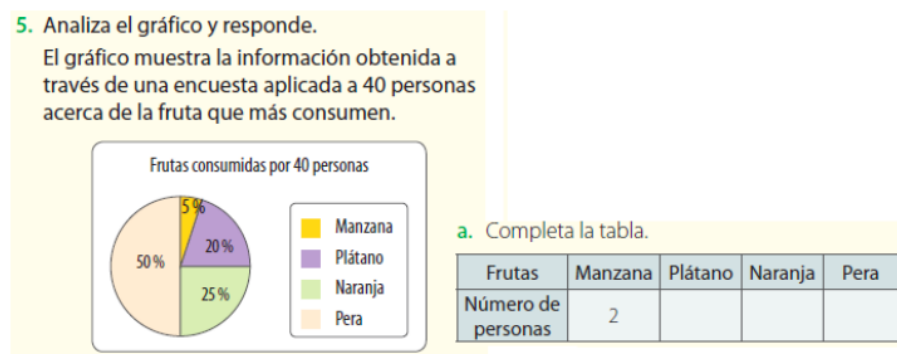

Figura 4 - Traducción de gráfico a tabla para $7^{\circ}$ Básico Fuente: Merino, Muñoz, Pérez, \& Rupin (2016, p. 348)

- SP4. Realizar una clasificación cruzada de dos variables. La tabla de doble entrada permite organizar la distribución de frecuencias de una variable estadística bidimensional, mediante tantas filas y columnas como modalidades presenten las variables que la conforman, cualitativas en el caso de tabla de contingencia. En cada cruce de modalidades de las respectivas variables, se representa la frecuencia conjunta (absoluta, relativa o porcentual) de los datos. Así mismo, destacamos la importancia de la tabla de doble entrada en el estudio de la asociación entre variables estadísticas bidimensionales (Ver Figura 3). Autor (2013) clasifican en tres grandes tipos las situaciones-problemas en estudios bidimensionales:

- SP4.1. Organización de la información de un conjunto de datos bivariados, es un aspecto importante en el estudio posterior de temas como la correlación y regresión (Autor, 2013). Correspondería al ejemplo mostrado en la Figura 3, puesto que para responder a las tareas planteadas, se requiere calcular los totales en la tabla que se presenta en la situación-problema.

- SP4.2. Análisis de las variables que conforman la variable estadística bidimensional. Cuando se analiza la variable estadística bidimensional es necesario delimitar cada una de las variables que la constituyen, como por ejemplo, la situación-problema que se muestra en la Figura 5, donde el estudiante posterior a describir las variables debe construir un gráfico apropiado para representar la información presentada en la tabla. En esta categoría se considera el análisis descriptivo de cada variable, los pasos requeridos para completar una tabla de doble entrada conocidas las medias marginales, así como el análisis de la dependencia funcional o estadística de las variables, su intensidad y sentido (Autor, 2014). 
DOI: $10.20396 /$ zet.v28i0.8656257

Define la variable en estudio en cada tabla y
representa en un gráfico adecuado de acuerdo
al tipo de variable.
a. Tabla 1: Número de funciones realizadas por
un grupo de teatro en Chile, Brasil y Argenti-
da entre el 2010 y 2012 .
\begin{tabular}{|c|c|c|c|}
\hline & 2010 & 2011 & 2012 \\
\hline Chile & 5 & 7 & 3 \\
\hline Brasil & 1 & 4 & 2 \\
\hline Argentina & 10 & 8 & 7 \\
\hline
\end{tabular}

Figura 5 - Análisis de las variables en una tabla de contingencia para $7^{\circ}$ Básico Fuente: Merino, Muñoz, Pérez, \& Rupin (2016, p. 361)

- SP4.3. Estudio de la regresión entre las variables que conforman la tabla, que persigue el análisis de la dependencia de las variables y, en el caso que existiera dependencia intensa, establecer un modelo de ajuste con fin predictivo (Autor, 2014). No encontramos ejemplos en los textos analizados.

Tabla 1 - Campos de problemas ligados a los diferentes tipos de tablas estadísticas

\begin{tabular}{|c|c|c|c|c|c|}
\hline \multirow[t]{2}{*}{ CAMPOS DE PROBLEMAS } & \multirow{2}{*}{$\begin{array}{l}\text { Tabla de } \\
\text { datos }\end{array}$} & \multicolumn{3}{|c|}{ Tabla de distribución } & \multirow{2}{*}{$\begin{array}{l}\text { Tabla de } \\
\text { contingencia }\end{array}$} \\
\hline & & Ord. & Ac. & Agr. & \\
\hline SP1. Resumen y organización & $\mathrm{X}$ & $\mathrm{X}$ & $x$ & $\mathrm{X}$ & 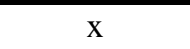 \\
\hline $\begin{array}{l}\text { SP2. Construcción de la distribución de una } \\
\text { variable }\end{array}$ & & $\mathrm{x}$ & $\mathrm{x}$ & $\mathrm{x}$ & \\
\hline SP3. Traducción de: & & & & & \\
\hline 1. Tabla a gráfico, o viceversa & $\mathrm{X}$ & $\mathrm{X}$ & $\mathrm{X}$ & $\mathrm{X}$ & $\mathrm{X}$ \\
\hline 2. Tabla a tabla & $\mathrm{X}$ & $\mathrm{x}$ & $\mathrm{x}$ & $\mathrm{x}$ & $\mathrm{x}$ \\
\hline 3. Tabla a texto, o viceversa & & $\mathrm{x}$ & $\mathrm{x}$ & $\mathrm{x}$ & $\mathrm{X}$ \\
\hline 4. Tabla a estadístico & $\mathrm{X}$ & $\mathrm{x}$ & $\mathrm{x}$ & $\mathrm{x}$ & $\mathrm{x}$ \\
\hline $\begin{array}{l}\text { SP4. Realizar una clasificación cruzada de } \\
\text { dos variables: }\end{array}$ & & & & & \\
\hline 1. Organización de la información & & & & & $\mathrm{X}$ \\
\hline 2. Análisis de las variables que conforman & & & & & $\mathrm{x}$ \\
\hline la variable estadística bidimensional & & & & & \\
\hline $\begin{array}{l}\text { 3. Estudio de la asociación y regresión entre } \\
\text { las variables que conforman la tabla. }\end{array}$ & & & & & $\mathrm{x}$ \\
\hline
\end{tabular}

Fuente: Elaborado por las autoras

En la Tabla 1 presentamos un resumen de las situaciones-problemas (SP) que implícita o explícitamente caracterizan a los tres tipos principales de tablas estadísticas. La Tabla de distribución se ha subdivido en tres categorías (ordinarias: Ord; acumuladas: Ac; datos agrupados: Agr) de acuerdo a los objetos matemáticos que en ellas intervienen, y los subniveles de complejidad semiótica, pero no hemos subdividido las tablas de contingencia al no haber encontrado en los libros de texto tablas de este tipo donde se utilicen intervalos de clase.

Podemos apreciar, que los tres primeros tipos de problema se pueden encontrar asociados a todos los tipos de tablas, por lo que pueden trabajarse estos campos de problema con cualquiera de ellas, mientras que el campo de problemas SP4, con sus diferentes subproblemas, sería propio de la tabla de contingencia.

\section{Lenguaje asociado al trabajo con tablas estadísticas}


El lenguaje permite representar un objeto matemático y, en relación a las tablas estadísticas, independiente de su tipo, se utilizan diferentes tipos de lenguaje matemático, tanto al trabajar con ellas como para comunicar el enunciado, procedimiento o la solución de una situación-problema. En resumen, identificamos la siguiente tipología de lenguaje, que es aplicable a todos los tipos de tablas:

- Verbal, presente en el título, modalidades de la variable (cuando es cualitativa), en las etiquetas señalando el tipo de frecuencias (e.g. absolutas, relativas, porcentajes) o etiquetas de última fila (totales). También incluye palabras que hacen referencia a las magnitudes representadas (por ejemplo, precios, alturas, distancias, etc.).

- Simbólico, dependiendo del tipo de tabla, pueden aparecer símbolos matemáticos en las etiquetas de los márgenes, filas superiores, inferiores, primera columna y última columna; en estos espacios aparecen símbolos, por ejemplo: $\mathrm{x}_{\mathrm{i}}$ (valor de una variable $\mathrm{X}) ; \mathrm{n}_{\mathrm{i}}$ (frecuencia absoluta); $\mathrm{f}_{\mathrm{i}}$ (frecuencia relativa), y operaciones como $\sum$ (sumatorio, con o sin subíndices).

- Numérico, en el cuerpo de datos aparecen diferentes tipos de números como enteros y racionales (decimales, fraccionarios, porcentajes).

- Diagramático. La propia tabla estadística se constituye en un posicionamiento espacial de una o dos dimensiones (derecha, izquierda, arriba, abajo, intersección, diagonal), a través de sus filas y columnas, lo que permite realizar transformaciones, combinaciones y construcciones, obedeciendo a ciertas reglas sintácticas o semánticas (Godino, Giacomone, Wilhelmi, Blanco, \& Contreras, 2015).

\section{Argumentos}

En cuanto a los argumentos, también comunes a las diferentes tablas, se utilizan para justificar la resolución de variadas situaciones-problemas, y también para comunicar los resultados obtenidos.

Las argumentaciones no sólo albergan las demostraciones formales deductivas, características de la matemática, sino cualquier enunciado utilizado para justificar (Godino, \& Recio, 2001). Por ejemplo, se pueden usar para validar si una serie de afirmaciones sobre la información expuesta en una tabla son verdaderas o falsas; interpretar en un determinado contexto, por qué interesa considerar una medida como la más representativa de una distribución presentada en una tabla, a partir del estudio de las medidas de tendencia central.

Los argumentos que son empleados en torno a la tabla vienen determinados por los conceptos, procedimientos y propiedades utilizados para dar respuesta a la situación-problema que se plantea. Así, encontramos diversidad de modos de argumentar como son (Recio, 1999):

- Ejemplos o contraejemplos. Concierne al empleo de ejemplos concretos para validar una determinada propiedad o procedimiento.

- Uso de representaciones gráficas desde una tabla, pues en ocasiones es útil representar gráficamente los datos de la tabla para argumentar un enunciado. 
DOI: $10.20396 /$ zet.v28i0.8656257

- $\quad$ Argumentos verbales deductivos, referidos a demostraciones deductivas, utilizando axiomas o teoremas que conforman dicha argumentación.

- Argumento algebraico deductivo, son aquellos que evidencian argumentos deductivos basados en lenguaje algebraico, asociados a la tabla. Se trata de manipulaciones algebraicas utilizadas para argumentar una propiedad, el empleo de alguna fórmula, etc.

\section{Conceptos y propiedades}

Un concepto matemático se reconoce por su definición y sus propiedades, las que pueden variar de acuerdo a la institución, lo que implica que tengan un carácter relativo. Las definiciones de conceptos son evocados por el estudiante cuando resuelve una situaciónproblema, por lo que es importante analizar el tratamiento de estos objetos en la enseñanza, pues la progresiva construcción de su significado incidirá en los conceptos que se expliquen y utilicen (Godino, 2017). En las tablas estadísticas, los conceptos y su profundidad obedecen al marco curricular (MINEDUC, 2015; 2018) de los niveles educativos al que se dirige nuestro estudio. Algunos de los conceptos ligados a las tablas que aparecen en las bases curriculares chilenas son: población, individuo, censo y muestras; variables y sus valores; intervalos de clase, extremos y marca de clase; distribución de frecuencias de variables estadísticas unidimensionales (absoluta, relativa, porcentual, acumulada); probabilidades; distribución de frecuencias de variables estadísticas bidimensionales (dobles, marginales, condicionales); además de otros conceptos como las medidas de centralización, posición y dispersión.

En la Tabla 2, se presenta un resumen de los conceptos que implícita o explícitamente caracterizan a los tres tipos principales de tablas estadísticas. Podemos observar la mayor complejidad de la tabla de distribución y de contingencia, respecto a la menor cantidad de conceptos que es necesario utilizar en el trabajo con la tabla de datos. De esta diversidad semiótica, se justifica también los niveles de complejidad que proponemos en el estudio de las tablas estadísticas.

Tabla 2 - Conceptos y propiedades ligados a los diferentes tipos de tablas estadísticas

\begin{tabular}{|c|c|c|c|c|c|}
\hline \multirow[t]{2}{*}{ CONCEPTOS } & \multirow{2}{*}{$\begin{array}{c}\text { Tabla } \\
\text { de datos }\end{array}$} & \multicolumn{3}{|c|}{ Tabla de distribución } & \multirow{2}{*}{$\begin{array}{c}\text { Tabla de } \\
\text { contingencia }\end{array}$} \\
\hline & & Ord. & Ac. & Agr. & \\
\hline Población, individuo & $\mathrm{x}$ & $\mathrm{x}$ & $\mathrm{x}$ & $\mathrm{x}$ & $\mathrm{x}$ \\
\hline Censo, muestra & $\mathrm{x}$ & $\mathrm{x}$ & $\mathrm{x}$ & $\mathrm{x}$ & $\mathrm{x}$ \\
\hline Variable, valores, & $\mathrm{x}$ & $\mathrm{x}$ & $\mathrm{x}$ & $\mathrm{x}$ & $\mathrm{x}$ \\
\hline Rango, máximo, mínimo & $\mathrm{x}$ & $\mathrm{x}$ & $\mathrm{x}$ & $\mathrm{x}$ & $\mathrm{x}$ \\
\hline Intervalos de clase, extremos, marca de clase & & & & $\mathrm{x}$ & $\mathrm{x}$ \\
\hline Frecuencias ordinarias & & $\mathrm{x}$ & $\mathrm{x}$ & $\mathrm{x}$ & $\mathrm{x}$ \\
\hline Frecuencia acumulada & & & $\mathrm{x}$ & $\mathrm{x}$ & \\
\hline Frecuencias dobles & & & & & $\mathrm{x}$ \\
\hline Frecuencias marginales y condicionadas & & & & & $\mathrm{x}$ \\
\hline Probabilidades simples & & $\mathrm{x}$ & $\mathrm{x}$ & $\mathrm{x}$ & $\mathrm{x}$ \\
\hline Probabilidades compuestas y condicionales & & & & & $\mathrm{x}$ \\
\hline Medidas de tendencia central, dispersión & $\mathrm{x}$ & $\mathrm{x}$ & $\mathrm{x}$ & $\mathrm{x}$ & $\mathrm{x}$ \\
\hline Covarianza, correlación, regresión & & & & & $\mathrm{x}$ \\
\hline PROPIEDADES & & & & & \\
\hline Tipos de variable y escala de medida & $\mathrm{x}$ & $\mathrm{x}$ & $\mathrm{x}$ & $\mathrm{x}$ & $\mathrm{x}$ \\
\hline
\end{tabular}




\begin{tabular}{|c|c|c|c|c|}
\hline $\begin{array}{l}\text { Suma de frecuencias absolutas y tamaño } \\
\text { muestra }\end{array}$ & $\mathrm{x}$ & & $\mathrm{x}$ & $\mathrm{x}$ \\
\hline Suma de frecuencias relativas & $\mathrm{x}$ & & $\mathrm{x}$ & $\mathrm{X}$ \\
\hline $\begin{array}{l}\text { Proporcionalidad de frecuencias relativas y } \\
\text { absolutas }\end{array}$ & $\mathrm{x}$ & & $\mathrm{x}$ & $\mathrm{x}$ \\
\hline $\begin{array}{l}\text { Valor creciente de las frecuencias acumuladas, } \\
\text { la última de las cuales es el tamaño muestral }\end{array}$ & & $\mathrm{x}$ & & \\
\hline $\begin{array}{l}\text { Relación entre frecuencias acumuladas y no } \\
\text { acumuladas en datos agrupados }\end{array}$ & & $\mathrm{x}$ & $\mathrm{x}$ & \\
\hline $\begin{array}{l}\text { Variable dependiente e independiente, } \\
\text { asociación entre variables }\end{array}$ & & & & $\mathrm{x}$ \\
\hline $\begin{array}{l}\text { Relación entre frecuencias relativas } \\
\text { condicionales, dobles y marginales }\end{array}$ & & & & $\mathrm{x}$ \\
\hline $\begin{array}{l}\text { Frecuencias esperadas en caso de } \\
\text { independencia }\end{array}$ & & & & $\mathrm{X}$ \\
\hline
\end{tabular}

Fuente: Elaborado por las autoras

En relación a las propiedades, estas se refieren a características específicas de los conceptos o relaciones entre los conceptos. Cada propiedad, se asocia a un objeto matemático o la relación entre objetos, por lo que regula su uso y contribuye al crecimiento del significado del objeto en cuestión (Godino, 2002; 2017). Para la tabla estadística (Ver Tabla 2), las principales propiedades se refieren a las relaciones entre tipos de frecuencia y dependen de si se trata de una distribución de frecuencias, o una tabla de contingencia y del tipo de frecuencias (acumuladas o no, en datos agrupados o no). Cabe destacar, que la tabla de contingencia abarca, prácticamente, la totalidad de propiedades, además de otras específicas de la misma. Esto evidencia su complejidad en cuanto al elevado nivel de comprensión que requiere el tratamiento de este tipo de representación tabular.

\section{Procedimientos utilizados en el trabajo con las tablas estadísticas}

Los procedimientos se refieren a los algoritmos o estrategias que se aplican en una tabla y permiten operar con los datos. Para las tablas identificamos tres procesos:

- Lectura de tabla, si la tabla se entrega construida y se piden algunos datos que deben obtenerse a partir de la misma, se requiere un proceso interpretativo de cada elemento que la conforma (título, etiquetas, valores) y de la tabla en su conjunto. Puede ser más o menos compleja la lectura, ya que se pueden considerar los niveles de lectura para los gráficos propuestos por Friel, Curcio y Bright (2001), que involucra cuatro procedimientos, los que se pueden trasladar para las tablas estadísticas: Leer los datos: la lectura de los datos se ajusta a lo mostrado explícitamente en la tabla, como leer una frecuencia para un valor determinado de la variable, o al contrario; Leer dentro de los datos: consiste en el uso de la información explícita de la tabla, por medio de cálculos sencillos con los datos para obtener información que no se encuentra, directamente, disponible en ella; Leer más allá de los datos: que implica inferir información que no se encuentra expuesta, ni es posible de extraer de forma aritmética; y Leer detrás de los datos: implica una valoración crítica del contenido de la tabla, como la fuente de la cual se ha extraído la información de la misma, además de relacionar dicha información con el contexto en que es planteado.

- Construcción de tablas. El estudiante debe formar la tabla a partir de los datos brutos. 
Duval (2003) indica que este es un procedimiento que implica el despliegue de una variedad de funciones cognitivas, necesarias para llevar a cabo esta tarea; se destaca, entre otras, la categorización de los datos y el cálculo de las frecuencias, cuando es necesario.

- $\quad$ Otros procedimientos, por ejemplo, las operaciones de recuentos de datos, cálculos de diferentes tipos de frecuencia, determinar estadísticos.

Además, podríamos considerar que la traducción entre representaciones implica tanto la lectura como la construcción.

Tabla 3 - Procedimientos ligados a los diferentes tipos de tablas estadísticas

\begin{tabular}{|c|c|c|c|c|c|}
\hline PROCEDIMIENTOS & \multirow{2}{*}{$\begin{array}{c}\text { Tabla de } \\
\text { datos }\end{array}$} & \multicolumn{3}{|c|}{ Tabla de distribución } & \multirow{2}{*}{$\begin{array}{c}\text { Tabla de } \\
\text { contingencia }\end{array}$} \\
\hline & & Ord. & Ac. & Agr. & \\
\hline Lectura de tablas & $\mathrm{x}$ & $\mathrm{X}$ & $\mathrm{x}$ & $\mathrm{x}$ & $\mathrm{x}$ \\
\hline Construcción de tablas & $\mathrm{x}$ & $\mathrm{x}$ & $\mathrm{x}$ & $\mathrm{x}$ & $\mathrm{x}$ \\
\hline Otros procedimientos & $\mathrm{x}$ & $\mathrm{x}$ & $\mathrm{x}$ & $\mathrm{x}$ & $\mathrm{x}$ \\
\hline
\end{tabular}

Fuente: Elaborado por las autoras

En la Tabla 3 podemos apreciar que los procedimientos, anteriormente descritos, son comunes para todos los tipos de tablas estadísticas. No obstante, se debe tener en cuenta, que cada una de estas acciones requiere de capacidades y destrezas específicas, dependiendo del tipo de representación tabular en cuestión, pues las tablas, aunque visualmente se vean similares, cada una posee diferente estructura que la caracteriza con distinto sentido y función.

\section{Distribución de los tipos de tablas en los textos analizados}

Para completar el trabajo, se ha realizado un análisis estadístico de la distribución de los diferentes tipos de tablas analizados en los textos de la muestra. La clasificación se ha dividido en los tres tipos de tablas estadísticas descritas anteriormente: tabla de datos, tabla de distribución de una variable y tabla de contingencia. A su vez, recordemos que la Tabla de distribución se ha subdivido en tres categorías: frecuencias ordinarias (absolutas, relativas y porcentuales), frecuencias acumuladas y distribución de la variable mediante datos agrupados.

Como resultado del análisis, observamos (Ver Tabla 4) que globalmente más de la mitad de las tablas presentes en los textos escolares corresponden a tablas de distribución, en primer lugar, las que muestran frecuencias ordinarias $(51,8 \%)$, seguido de las tablas de datos $(19,2 \%)$, luego las tablas de contingencia $(13,1 \%)$. Las de distribución de frecuencias de datos agrupados $(3,9 \%)$ son las que menos se plantean en los textos, así como de frecuencias acumuladas (12\%). La tendencia de nuestros resultados es similar a lo evidenciado en los estudios de Díaz-Levicoy y colaboradores (2015; 2017), a pesar de que en las citadas investigaciones sólo aparecen las tablas de frecuencias ordinarias, debido a la menor edad de los estudiantes. En su estudio, la mayoría de las tablas son de conteo, ya desatendidas en los cursos que hemos analizado. La tabla de frecuencia aparece alrededor del $32 \%$ en los cursos de tercer grado y no hay presencia de la tabla de doble entrada, aunque sí se encuentra en contadas ocasiones de los cursos $1^{\circ}$ y $2^{\circ}$ de Educación Básica. En nuestro estudio, también pudimos detectar una escasa presencia de la tabla de contingencia, a pesar de que aparece en todos los niveles explorados y, paradójicamente pese a su complejidad, se utiliza más en los 
niveles inferiores.

Tabla 4 - Frecuencia (y porcentaje) del tipo de tabla, según nivel educativo

\begin{tabular}{|c|c|c|c|c|c|c|}
\hline TIPO DE T & BLA & $5^{\circ} \mathrm{EB}$ & $6^{\circ} \mathrm{EB}$ & $7^{\circ} \mathrm{EB}$ & $8^{\circ} \mathrm{EB}$ & Total \\
\hline \multicolumn{2}{|c|}{ Tabla de datos } & $27(18,1)$ & $9(8)$ & $65(14,3)$ & $90(32,5)$ & $191(19,2)$ \\
\hline \multirow{3}{*}{$\begin{array}{l}\text { Tabla de } \\
\text { distribución }\end{array}$} & Ordinarias & $81(54,4)$ & $83(73,4)$ & $272(59,9)$ & $78(28,1)$ & $514(51,8)$ \\
\hline & Acumuladas & & & $77(17)$ & $42(15,2)$ & $119(12)$ \\
\hline & Agrupados & & & & $39(14,1)$ & $39(3,9)$ \\
\hline \multicolumn{2}{|c|}{ Tabla de contingencia } & $41(27,5)$ & $21(18,6)$ & $40(8,8)$ & $28(10,1)$ & $130(13,1)$ \\
\hline \multicolumn{2}{|l|}{ Total } & 149 & 113 & 454 & 277 & 993 \\
\hline
\end{tabular}

Fuente. Elaborado por las autoras

Observamos también variación con el nivel escolar. En los niveles de $5^{\circ}$ y $6^{\circ}$ curso, el estudio con las tablas se centra, principalmente, en las de distribución con frecuencias ordinarias. A partir de $7^{\circ}$ curso, aparecen las tablas estadísticas que incorporan las frecuencias acumuladas, y en un porcentaje muy bajo en $8^{\circ}$ curso se estudia la distribución de frecuencias con datos agrupados en intervalos. También observamos, que el $5^{\circ}$ nivel es el que muestra una mayor presencia de las tablas de contingencia $(27,5 \%)$.

\section{Conclusiones}

En la exposición realizada, se puede apreciar la complejidad cognitiva ligada en torno a la tabla estadística, la que implica la comprensión de una variedad de objetos matemáticos necesarios de poner en juego para llevar a cabo con éxito tareas que las involucren. Cada uno de los objetos matemáticos, tendrá diferentes niveles de formalización conforme se avance de nivel educativo, pues no es lo mismo tratar con una tabla estadística en $5^{\circ}$ curso que en $8^{\circ}$ curso, donde las tareas requieren de mayor conocimiento y destrezas matemáticas.

El análisis semiótico de la tabla estadística, a través de los elementos primarios del enfoque ontosemiótico (Godino, 2017), se convierte en una herramienta metodológica útil para profundizar e identificar los diversos objetos matemáticos que se requiere conocer para trabajar con los diferentes tipos de tablas propuestos por Lahanier-Reuter (2003), dado que cada una de ellas posee una estructura y función específica, logrando de este modo, caracterizar el significado institucional pretendido de la tabla estadística.

El estudio de la distribución de las tablas en los textos analizados, permite detectar que las tablas de contingencia, del mayor nivel de complejidad semiótico (N4), son las que menos aparecen en los textos, hecho que llama la atención dado que este tipo de tablas es la que presenta una mayor riqueza de objetos matemáticos y relaciones posibles de establecer en ella, siendo mayoritariamente su uso en $5^{\circ}$ curso y con una distribución decreciente a medida que se incrementa el nivel educativo. Por otra parte, la mayoría de las actividades analizadas se basan en tablas de nivel de complejidad N3.

Con este estudio, confirmamos la aplicabilidad de los niveles de complejidad, propuesto inicialmente por Arteaga (2011) para los gráficos estadísticos a las tablas. Para ello, se realizó un ajuste de los niveles, donde cada uno de ellos fue asociado a un tipo de tabla estadística. Esto con el fin de profundizar y describir de mejor manera la complejidad semiótica, 
especialmente los niveles $\mathrm{N} 3$ y N4, lo que ha ofrecido una complementación del modelo propuesto por Arteaga, proponiendo tres subniveles de complejidad para N3, y dos subniveles para el mayor grado de complejidad N4, que obedecen a los distintos tipos de objetos matemáticos que es posible identificar en la tabla, tales como la consideración de las frecuencias ordinarias o acumuladas y los intervalos de clase, lo que posibilita un mayor grado de dificultad, dentro de un mismo nivel de complejidad semiótico. De este modo, se ofrece un enriquecimiento de los niveles de complejidad asociados a las representaciones estadísticas.

\section{Agradecimentos:}

EDU2016-74848-P (AEI, FEDER), Grupo de Investigación FQM-126 (Junta de Andalucía) y Beca CONICYT Folio: 72190280

\section{Referencias}

Arteaga, P. (2011). Evaluación de conocimientos sobre gráficos estadísticos y conocimientos didácticos de futuros profesores (Tesis doctoral). Universidad de Granada, Granada, España.

Chick, H. (2004). Tools for transnumeration: Early stages in the art of data representation. In I. Putt, R. Faragher, \& M. McLean (Eds.), Mathematics education for the third millennium: Towards 2010, Proceedings of the Twenty-seventh Annual Conference of the Mathematics Education Research Group of Australasia (pp.167-174). Sydney: MERGA.

Díaz-Levicoy, D., Morales, R., \& López-Martín, M. M. (2015). Tablas estadísticas en libros de texto chilenos de $1^{\circ}$ y $2^{\circ}$ año de Educación Primaria. Revista Paranaense de Educação Matemática, 4(7), 10-39.

Díaz-Levicoy, D., Ruz, F., \& Molina-Portillo, E. (2017). Tablas estadísticas en libros de texto chilenos de tercer año de Educación Primaria. Espaço Plural, 18(36), 196-218.

Duval, R. (2003). Comment analyser le fonctionnement représentationnel des tableaux et leur diversité ? Spirale-Revue de recherches en éducation, 32(32), 7-31.

Engel, J. (2019). Statistical literacy and society. En J. M. Contreras, M. M. Gea, M. M. LópezMartín, \& E. Molina-Portillo (Eds.), Actas del Tercer Congreso Internacional Virtual de Educación Estadística. Granada: Grupo FQM-126. Disponible en: www.ugr.es/local/fqm126/civeest.html.

Estrella, S. (2014). El formato tabular: una revisión de literatura. Revista Actualidades Investigativas en Educación, 14(2), 1-23.

Feinberg, R., \& Wainer, H. (2012) Extracting sunbeams from cucumbers. Journal of Computational and Graphical Statistics, 20(4), 793-810.

Franklin, C., Kader, G., Mewborn, D., Moreno, J., Peck, R., Perry, M., \& Scheaffer, R. (2005). Guidelines for assessment and instruction in statistics education (GAISE) report: A PreK- 12 curriculum framework. Alexandria, VA: American Statistical Association. Disponible en: www.amstat.org/Education/gaise/.

Friel, S., Curcio, F., \& Bright, G. (2001). Making sense of graphs: critical factors influencing 
DOI: $10.20396 /$ zet.v28i0.8656257

comprehension and instructional implications. Journal for Research in mathematics Education, 32(2), 124-158.

Gal, I. (2002). Adults' statistical literacy: Meanings, components, responsibilities. International statistical review, 70(1), 1-25.

Godino, J. D. (2002). Un enfoque ontológico y semiótico de la cognición matemática. Recherches en Didactiques des Mathematiques, 22(2-3), 237-284.

Godino, J. D. (2017). Construyendo un sistema modular e inclusivo de herramientas teóricas para la educación matemática. En J. M. Contreras, P. Arteaga, G. R. Cañadas, M. M. Gea, B. Giacomone, \& M. M. López-Martín (Eds.), Actas del Segundo Congreso International Virtual sobre el Enfoque Ontosemiótico del Conocimiento y la Instrucción Matemática. Disponible en: http://enfoqueontosemiotico.ugr.es/civeos/godino.pdf.

Godino, J. D., Giacomone, B., Wilhelmi, M. R., Blanco, T. F., \& Contreras, A. (2015). Configuraciones de prácticas, objetos y procesos imbricadas en la visualización espacial y el razonamiento diagramático. Departamento de Didáctica de la Matemática. Universidad de Granada, España.

Godino, J. D., \& Recio, Á. M. (2001). Significados institucionales de la demostración. Implicaciones para la educación matemática. Enseñanza de las ciencias: Revista de investigación y experiencias didácticas, 19(3), 405-414.

Koschat, M. (2005). A case for simple tables. The American Statistician, 59(1), 31-40.

Lahanier-Reuter, D. (2003). Différents types de tableaux dans l'enseignement des statistiques. Spirale-Revue de recherches en éducation, 32(32), 143-154.

Maldonado, L., \& Castro, C. (2017). Texto del estudiante Matemática $6^{o}$ básico. Santiago: Grupo Santillana de ediciones.

Mayring, P. (2000). Qualitative inhalts analyse. Forum: Qualitative Sozialforschung/Forum: Qualitative Social Research, 1(2). Art. 20.

Merino, R., Muñoz, V., Pérez, B., \& Rupin, P. (2016). Texto del estudiante Matemática $7^{o}$ básico. Santiago: Ediciones SM.

MECD (2014). Real Decreto 126/2014, de 28 de febrero, por el que se establece el currículo básico de la Educación Primaria. Madrid, España: Autor.

MINEDUC (2013). Matemática. Programa de Estudio Quinto Año Básico. Santiago, Chile: Unidad de currículum y evaluación.

MINEDUC (2015). Bases curriculares $7^{\circ}$ Básico a $2^{\circ}$ Medio. Santiago, Chile: Unidad de currículum y evaluación.

MINEDUC (2018). Bases Curriculares Primero a Sexto Básico. Santiago, Chile: Unidad de currículum y evaluación.

NCTM (2000). Principles and standards for school mathematics. Reston, VA: National Council of Teachers of Mathematics.

Recio, A. M. (1999). Una aproximación epistemológica a la enseñanza y aprendizaje de la demostración matemática (Tesis doctoral). Universidad de Granada, Granada, España.

Wild, C., \& Pfannkuch, M. (1999). Statistical thinking in empirical enquiry. International 
Statistical Review, 67(3), 223-248.

\section{ANEXO}

Libros utilizados en el análisis: $5^{\circ}$ Curso.

Kheong, F. H., Soon, G. K., \& Ramakrishnan, C. (2017). Texto del estudiante Matemática $5^{o}$ básico. Santiago: Marshall Cavendish Education.

Kheong, F. H., Soon, G. K., \& Ramakrishnan, C. (2017). Cuaderno de ejercicios Matemática $5^{\circ}$ básico. Santiago: Marshall Cavendish Education.

Kheong, F. H., Soon, G. K., \& Ramakrishnan, C. (2017). Guía didáctica del docente - Tomo 2 Matemática $5^{\circ}$ básico. Santiago: Marshall Cavendish Education.

Libros utilizados en el análisis: $6^{\circ}$ Curso.

Maldonado, L., \& Castro, C. (2017). Texto del estudiante Matemática 6º́ básico. Santiago: Grupo Santillana de ediciones.

Castro, C. (2017). Cuaderno de ejercicios Matemática $6^{\circ}$ básico. Santiago: Grupo Santillana de ediciones.

Juillet, I., \& Martínez, M. (2017). Guía didáctica del docente - Tomo 2 Matemática $6^{o}$ básico. Santiago: Grupo Santillana de ediciones.

Libros utilizados en el análisis: $7^{\circ}$ Curso.

Merino, R., Muñoz, V., Pérez, B., \& Rupin, P. (2016). Texto del estudiante Matemática $7^{o}$ básico. Santiago: Ediciones SM.

Santis, M. (2016). Cuaderno de ejercicios Matemática $7^{\circ}$ básico. Santiago: Ediciones SM. Raydoret del Valle, J. (2016). Guía didáctica del docente Matemática $7^{\circ}$ básico. Santiago: Ediciones SM.

Libros utilizados en el análisis: $8^{\circ}$ Curso.

Catalán, D., Pérez, B., Prieto, C., \& Rupin, P. (2016). Texto del estudiante Matemática $8^{o}$ básico. Santiago: Ediciones SM.

Muñoz, V., \& Chacón, A. (2016). Cuaderno de ejercicios Matemática $8^{\circ}$ básico. Santiago: Ediciones SM.

Muñoz, V., \& Manosalva, C. (2016). Guía didáctica del docente Matemática $8^{\circ}$ básico. Santiago: Ediciones SM. 